\title{
Serum Level of Calprotectin as a Potential Marker of Inflammation in Acne Vulgaris Diagnosis and Severity Estimation \\ Mohammed A. Basha ${ }^{1}$, Rana G. Abdelmageed ${ }^{2}$, Noha Rabie Bayomy ${ }^{* 1}$ \\ Departments of ${ }^{1}$ Dermatology \& Andrology Department and ${ }^{2}$ Medical Biochemistry \& Molecular Biology, Faculty of Medicine, Menoufia University, Shebin El Kom, Egypt. \\ *Corresponding author: Rana G. Abdelmageed, Mobile: (+20) 01099522518, E-Mail: ranaagaamal@gmail.com
}

\begin{abstract}
Background: Acne vulgaris (AV) is a common inflammatory skin disease involving dysfunction of the pilosebaceous unit. Many mechanisms for the pathogenesis of acne have been postulated. However, the precise pathogenesis is still uncertain.

Objective: This study aimed to measure serum calprotectin levels in AV and to correlate its level with the activity and severity of the disease.

Patients and methods: A total of 80 subjects were subdivided into two groups; group I included 40 patients complaining of AV with different severity levels, and group II had 40 healthy age and sex-matched participants as a control group. Serum calprotectin level was measured in both groups by the enzyme-linked immunosorbent assay (ELISA) method.

Results: Serum calprotectin concentration was statistically significantly $(\mathrm{p}=0.0001)$ higher in the patient group when compared to the controls. There was a statistically significant $(\mathrm{p}=0.001)$ difference among subgroups of patients according to disease severity regarding serum calprotectin concentration. There was a high-significant positive correlation between serum calprotectin concentration and disease severity. Calprotectin concentration could be used to diagnose AV with cutoff points higher than $1.03 \mathrm{ng} / \mathrm{ml}$ (with $77.5 \%$ sensitivity, $80 \%$ specificity) and also can detect its severity with cutoff points higher than $1.1 \mathrm{ng} / \mathrm{ml}$ with $96.3 \%$ sensitivity, $100 \%$ specificity to differentiate the mild cases from moderate and severe ones.

Conclusion: Serum calprotectin concentration represents a valuable tool for diagnosing the inflammatory nature and monitoring the disease activity in AV patients.
\end{abstract}

Keywords: Acne vulgaris, Calprotectin.

\section{INTRODUCTION}

Acne vulgaris is a common cutaneous chronic inflammatory disorder with complex pathogenesis. Four factors play vital roles in acne pathophysiology: androgen-induced hyper seborrhea and dysseborrhea, altered keratinization of the pilosebaceous duct, cutibacterium acnes (P. acnes), and inflammation (1). The development of acne is mainly driven by increased sebum production, increased proliferation, and reduced desquamation of keratinocytes in the pilosebaceous unit. As sebum and keratinocytes hold together, the keratotic plug gradually forms and obstructs the pilosebaceous ducts, finally creating micro comedones (2) Bacterial colonization by Cutibacterium acnes aggravates the course of the disease in various manners, but its role as a prerequisite of the induction of acne is disputable ${ }^{(3)}$. Acne rates of prevalence in the range of $41.7 \%$ to $93.3 \%$ were reported in different countries with populations aged 12 to 18 . Acne prevalence can vary from report to other because of methodological differences such as definition grading of acne and population characteristics ${ }^{(4)}$.

Calprotectin is one of the family members of S100 proteins with two subunits (S100A8 and S100A9), isolated primarily from granulocytes and monocytes. Calprotectin is released from activated neutrophils, and it can be identified in serum or other body fluids like urine, feces, cerebrospinal fluid, synovial fluid, or saliva. Therefore, it has been suggested to serve as an inflammatory marker in many disorders ${ }^{(5)}$. Calprotectin subunits genes are encoded on chromosome 1q21, containing a cassette of genes known as the epidermal differentiation complex (EDC). This complex is involved in various biological processes, including the regulation of terminal cellular differentiation through a series of coordinated and interdependent signaling pathways. Moreover, it is a very sensitive diagnostic biomarker for many inflammatory diseases and may also be represented as a target of many therapies, which may give S100A8/ A9 clinical significance in many diseases ${ }^{(\boldsymbol{6})}$.

S100A8/A9 complex has a broad range of intracellular and extracellular biological functions. Intracellular functions through the participation in cytoskeleton modulation, antibacterial activities when released to the extracellular space, S100A8/A9 can play a crucial role in stimulating leukocyte recruitment and secretion of cytokines responsible for sustainment exacerbation of inflammation ${ }^{(7)}$. Furthermore, S100A8/A9 has been demonstrated as an endogenous agonist of toll-like receptor 4 (TLR4). On binding to TLR4 and the receptor for advanced glycation end products (RAGE), it can mediate the extracellular functions of S100A8/A9 ${ }^{(8)}$. Calprotectin is strongly expressed in infiltrating inflammatory cells, and it can be found in almost all dermatoses associated with the hyperproliferation of epithelial cells ${ }^{(\mathbf{9})}$. 
This study aimed to measure the level of serum calprotectin in $\mathrm{AV}$ and to correlate its level with the activity and severity of the disease.

\section{SUBJECTS AND METHODS}

This case-control study was conducted on patients selected from the Outpatient Clinic of Dermatology \& venerology department of Menoufia University Hospitals and Berket Elsaba General Hospital. The 80 subjects of the present study were divided into two groups: Group 1 included 40 patients with AV with the three degrees, mild, moderate, and severe with a mean age of $20.1 \pm 2.9$ years. Regarding sex, $15(37.5 \%)$ were males, and $25(62.5 \%)$ were females. Group 2 included 40 age and sex-matched healthy subjects as controls with a mean age of $20.9 \pm$ 3.2 years [20 (50\%) were males, and $20(50 \%)$ were females].

Inclusion criteria: Patients with AV with the three degrees, mild, moderate, and severe, were included based on the number of inflammatory eruptions on half of the face as 0-5, mild; 6-20, moderate; 21-50, severe; and more than 50 , very severe ${ }^{(\mathbf{1 0})}$.

Exclusion criteria: Patients with psoriatic arthritis, rheumatoid arthritis, inflammatory bowel disease, gastrointestinal diseases, multiple sclerosis and other cutaneous diseases than acne.

Each case was subjected to complete history taking including the age of onset, family history of acne vulgaris, and history of drug intake, complete general examination, and dermatological examination. Laboratory investigation included venous blood samples that was taken from all participants on a plain tube under complete aseptic conditions for serum separation. The blood was allowed to clot for 20 minutes then centrifuged for 10 minutes at 4000 r.p.m. for serum separation. The serum was immediately frozen at $-80^{\circ} \mathrm{C}$ until analysis. Serum calprotectin was determined by the ELISA kit provided by the Sun Red Bio (Shanghai-China).

Ethical approval:

Informed written consent was obtained from all participants after being informed about the aim and process of the study. The Local Research Ethics Committee of Menoufia Faculty of Medicine approved the study design with approval number 3/2020 DERM5. This work has been carried out in accordance with The Code of Ethics of the World Medical Association (Declaration of Helsinki) for studies involving humans.

\section{Statistical analysis:}

The results were statistically analyzed by SPSS version 20.0 (SPSS Inc., Chicago, IL, USA). Statistics were calculated in terms of number and percentage for qualitative data. Mean and standard deviation (SD) was used for the quantitative continues group. Tests used for significant difference were Chi-square test (X2) for qualitative variable and Student $\mathrm{t}$-test to compare the mean and SD of 2 sets of quantitative data. One-way analysis of Variance (ANOVA) test was used to compare three or more groups having quantitative normally distributed data. Pearson's correlation was used to study the correlation between two variables. Spearman correlation (r) was used to evaluates the linear association between 2 nonparametric quantitative variables. Sensitivity, specificity, positive predictive value (PPV), negative predictive value (NPV), and Receiver Operating Characteristic curve (ROC Curve) to illustrate the diagnostic ability of a binary classifier system as its discrimination threshold is varied. The significance level was set at a p-value less than or equal to 0.05 .

\section{RESULTS}

There was no significant statistical difference between groups regarding age, sex, and BMI. The age of patients and controls were $20.1 \pm 2.9$ years and 20.9 \pm 3.2 years, respectively. Among the AV patients, 15 (37.5\%) were males, and $25(62.5 \%)$ were females, whereas $20(50 \%)$ members of the control group were males and $20(50 \%)$ were females. The occupation of control subjects, 12 were students, 16 were housewives, and 12 were workers. However, in AV subjects, students were 14 , housewives were 16 , and workers were 10 (Table 1).

Table (1): Characteristics of studied groups ( $\mathrm{n}=80)$

\begin{tabular}{|c|c|c|c|c|}
\hline Characteristics & Patients $(n=40)$ & Control $(n=40)$ & Test & P value \\
\hline $\begin{array}{l}\text { Age } \\
\text { Mean } \pm \text { SD } \\
\text { range }\end{array}$ & $\begin{array}{c}20.1 \pm 2.9 \\
18-24\end{array}$ & $\begin{array}{c}20.9 \pm 3.2 \\
18-26\end{array}$ & T-test $=1.2$ & $0 . .2$ \\
\hline Sex: & $\mathbf{N}$ & $\%$ & \multirow[b]{2}{*}{$\begin{array}{c}\chi^{2} \text { test }= \\
1.2\end{array}$} & \multirow[b]{2}{*}{0.3} \\
\hline $\begin{array}{l}\text { Male } \\
\text { Female }\end{array}$ & $\begin{array}{l}15(37.5 \%) \\
25(62.5 \%)\end{array}$ & $\begin{array}{l}20(50 \%) \\
20(50 \%)\end{array}$ & & \\
\hline $\begin{array}{l}\text { Occupation: } \\
\text { Student } \\
\text { Housewife } \\
\text { Worker }\end{array}$ & $\begin{array}{l}14(35 \%) \\
16(40 \%) \\
10(25 \%)\end{array}$ & $\begin{array}{l}12(30 \%) \\
16(40 \%) \\
12(30 \%)\end{array}$ & $\begin{array}{c}\text { Likehood } \\
\text { ratio= } \\
0.34\end{array}$ & 0.8 \\
\hline
\end{tabular}


Concerning the clinical characters of the 40 patients, $29(72.5 \%)$ had a stationary course, and 11 $(27.5 \%)$ had a progressive course. The mean duration of the disease course was $3.25 \pm 1.1$ years. Regarding $72.5 \%$ who had a stationary course, half $(50 \%)$ of them had AV in their face, but one-fifth (10\%) had AV in the face and chest. Four-fifths (80\%) had no acne scar. About one-third (35\%) had severe AV, while about onethird (32.5\%) had mild and moderate AV for each. Out of the 40 patients, $60 \%$ had a positive history, $1.24 \pm$ 0.43 had a positive family history, and $1.15 \pm 0.19$ had a positive smoking history (Table 2).

Table (2): Severity of AV in the case group $(\mathrm{No}=40)$

\begin{tabular}{|l|c|c|}
\hline Variable & No=40 & \% \\
\hline Course: & 29 & 72.5 \\
Stationary & 11 & 27.5 \\
Progressive & 20 & 50 \\
\hline Site: & 5 & 12.5 \\
Face & 4 & 10 \\
Face/back & 11 & 27.5 \\
Face /chest & & \\
Face /chest/back & 32 & 80 \\
\hline Acne scar: & 8 & 20 \\
Absent & 13 & 32.5 \\
Present & 13 & 32.5 \\
\hline Grade: & 14 & 35 \\
Mild & \multicolumn{2}{|c|}{ Mean \pm SD:3.25 \pm 1.1} \\
Moderate & \multicolumn{2}{|c|}{ Range: $1-5$ years } \\
Severe & \multicolumn{2}{|c|}{} \\
\hline Duration of the & \multicolumn{2}{|c|}{} \\
disease &
\end{tabular}

In $\mathrm{AV}$ patients, serum calprotectin concentration was $1.21 \pm 0.34$, while in the controls, serum calprotectin concentration was $0.98 \pm 0.06$. Thus, there was a significant $(\mathrm{p}=0.0001)$ statistical difference between groups regarding serum calprotectin concentration (Table 3).

Table (3): Comparing mean of calprotectin serum level in patients and controls

\begin{tabular}{|l|c|c|c|c|}
\hline & $\begin{array}{c}\text { Patients } \\
(\mathbf{N o = 4 0 )})\end{array}$ & $\begin{array}{c}\text { Controls } \\
(\mathbf{N o =}=\mathbf{4 0})\end{array}$ & $\begin{array}{c}\text { Student- } \\
\text { T test }\end{array}$ & P-value \\
\hline Calproctin & $1.21 \pm$ & $0.98 \pm$ & 4.14 & $0.0001^{*}$ \\
level & 0.34 & 0.06 & & \\
\hline
\end{tabular}

There was no significant correlation between calprotectin serum level and BMI \& duration of the disease $(p>0.05)$. There was no statistically significant difference between those with a family history of AV\& those without regarding calprotectin serum level ( $p$ > $0.05)$.

There was no statistical difference between those who have a history of smoking and those who did not smoke regarding calprotectin serum level $(\mathrm{p}>0.05)$. There was no significant statistical difference between AV's stationary or progressive course regarding calprotectin serum level ( $\mathrm{p}>0.05$ ) (Tables 4, $5 \& 6$ ).
Table (4): Correlation of calprotectin level and BMI \& duration of the disease

\begin{tabular}{|l|c|c|}
\hline \multirow{2}{*}{ Variable } & \multicolumn{2}{c|}{ Calprotectin level } \\
\cline { 2 - 3 } & $\mathbf{R}$ & P-value \\
\hline BMI & 0.05 & 0.6 \\
\hline Duration of the disease & 0.02 & 0.8 \\
\hline
\end{tabular}

Table (5): Correlating the calprotectin serum level with a history of smoking $\&$ family history variables

\begin{tabular}{|l|c|c|c|}
\hline & Mean \pm SD & T-test & P-value \\
\hline Family history: & & & \\
Negative & $1.16 \pm 0.16$ & -0.74 & 0.5 \\
Positive & $1.24 \pm 0.33$ & & \\
\hline $\begin{array}{l}\text { History of smoking: } \\
\text { Negative } \\
\text { Positive }\end{array}$ & $1.21 \pm 0.36$ & 0.3 & 0.7 \\
\hline
\end{tabular}

Table (6): Calprotecting serum level and onset \& course of acne

\begin{tabular}{|l|c|c|c|}
\hline Variable & Mean \pm SD & T-test & $\begin{array}{c}\text { P- } \\
\text { value }\end{array}$ \\
\hline Course: & $1.24 \pm 0.39$ & 1.08 & 0.3 \\
Stationary & $1.11 \pm 0.16$ & & \\
Progressive & \\
\hline
\end{tabular}

There was a highly significant $(\mathrm{p}=0.001)$ statically difference among patients' subgroups of severity regarding serum calprotectin concentration [ $0.98 \pm$ $0.06,1.16 \pm 0.05$, and $1.46 \pm 0.48$ for mild, moderate, and severe cases, respectively] as shown in Table (7).

Table (7): Comparing mean of calproctein serum level between grades of acne

\begin{tabular}{|l|c|c|c|}
\hline Grade & Mean \pm SD & F-Test & P-value \\
\hline Mild & $0.98 \pm 0.06$ & & \\
Moderate & $1.16 \pm 0.05$ & 9.3 & $0.001^{*}$ \\
\hline Severe & $1.46 \pm 0.38$ & & \\
\hline
\end{tabular}
There was a positive correlation between
calprotectin serum level and grade of acne (p-value <
0.05) $(\mathrm{R}=0.9)$ as shown in table (8).

Table (8): Sperman correlation of calprotectin serum level and AV grade

\begin{tabular}{|l|c|c|}
\hline \multirow{2}{*}{ Variable } & \multicolumn{2}{|c|}{ Calprotectin level } \\
\cline { 2 - 3 } & R & P-value \\
\hline AV grade & +0.9 & $0.0001^{*}$ \\
\hline
\end{tabular}

This study demonstrated that serum calprotectin could diagnose $\mathrm{AV}$ cases at a cutoff point higher than $1.03(\mathrm{ng} / \mathrm{ml})$ with $77.5 \%$ sensitivity, $80 \%$ specificity, $79.5 \%$ PPV, $78 \%$ NPV, and accuracy of $78.75 \%$. While, detecting AV severity using mild cases versus moderate and severe cases, the cutoff point higher than 1.1 (ng/ ml) had $96.3 \%$ sensitivity, $100 \%$ specificity, $100 \%$ PPV, $92.8 \%$ NPV, and accuracy of $97.5 \%$. While using mild and moderate cases versus severe cases, the cutoff point higher than $1.22(\mathrm{ng} / \mathrm{ml})$ had $92.9 \%$ sensitivity, $100 \%$ specificity, $100 \%$ positive predictive value, $96.3 \%$ negative predictive value, and accuracy of $97.5 \%$ (Table 9 and figures $1,2 \& 3$ ). 
Table (9): ROC curve for calprotectin serum level for A) diagnosis of AV, B) for diagnosis of severity of AV (mild versus moderate and severe), C) diagnose the severity of AV (mild and moderate versus severe).

\begin{tabular}{|c|c|c|c|c|c|c|c|}
\hline & Best cut off point & AUC & Sensitivity & specificity & PPV & NPV & Accuracy \\
\hline A & $\mathbf{1 . 0 3}$ & $\mathbf{0 . 8 3}$ & $\mathbf{7 7 . 5 \%}$ & $\mathbf{8 0 \%}$ & $\mathbf{7 9 . 5 \%}$ & $\mathbf{7 8 \%}$ & $78.75 \%$ \\
\hline B & $\mathbf{1 . 1}$ & $\mathbf{0 . 9 9}$ & $\mathbf{9 6 . 3 \%}$ & $\mathbf{1 0 0 \%}$ & $\mathbf{1 0 0 \%}$ & $\mathbf{9 2 . 8}$ & $97.5 \%$ \\
\hline C & 1.22 & 0.98 & $92.9 \%$ & $100 \%$ & $100 \%$ & $96.3 \%$ & $97.5 \%$ \\
\hline
\end{tabular}

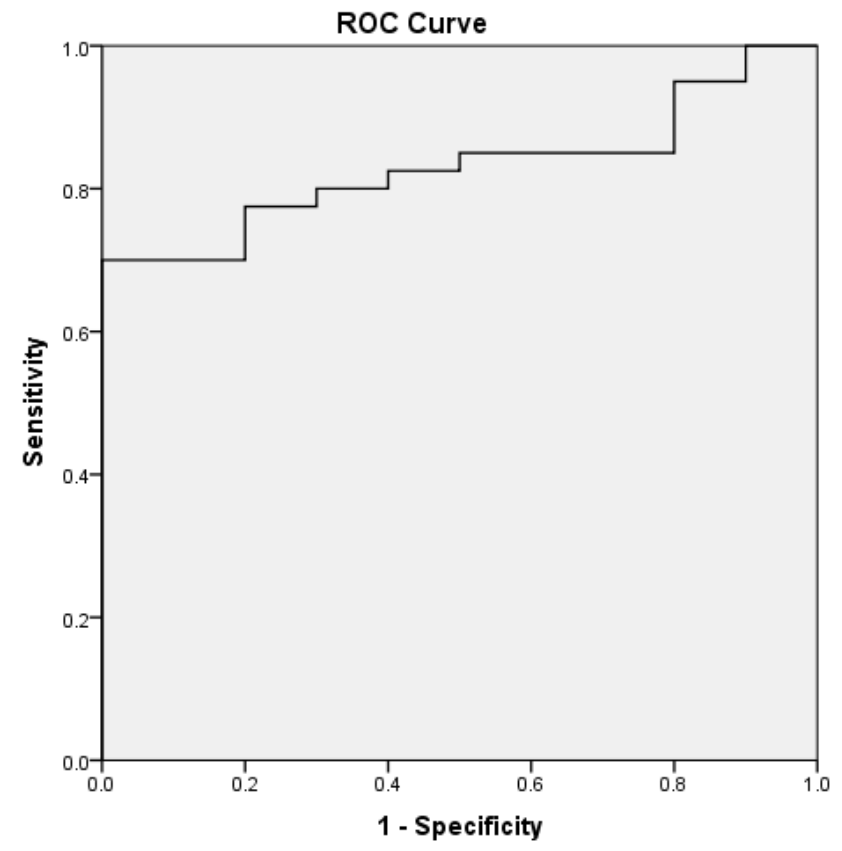

Fig. (1): ROC curve for calprotectin serum level for diagnosis of AV.

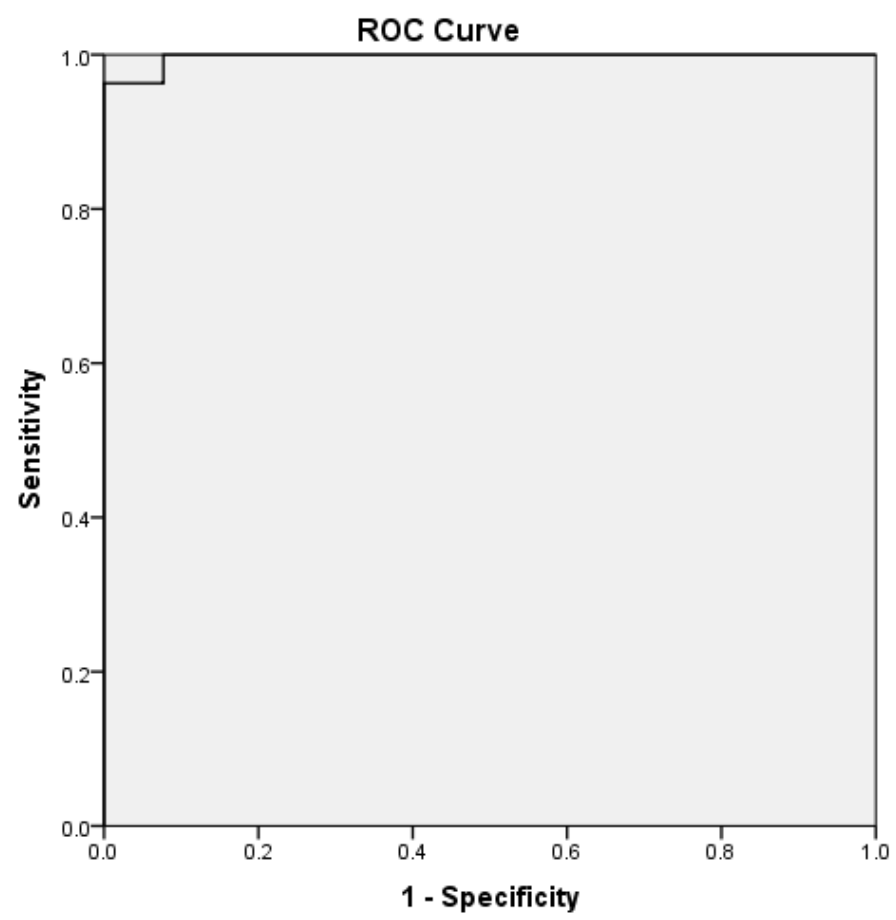

Fig. (2): ROC curve for calprotectin serum level for diagnosis of severity of AV (mild versus moderate and severe). 


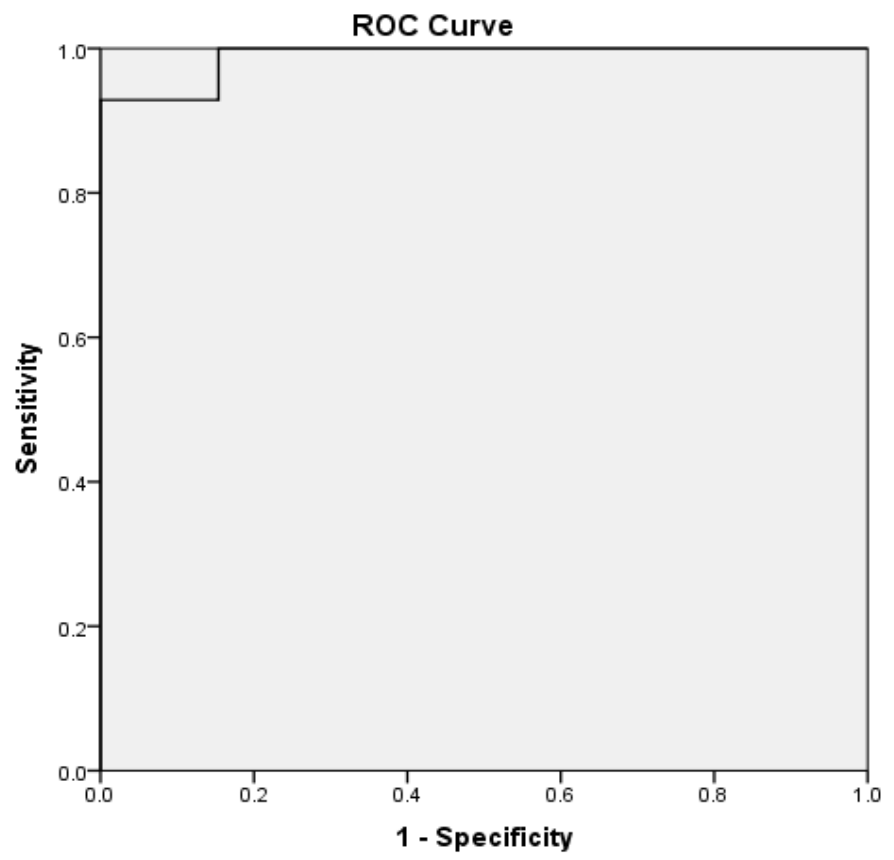

Fig. (3): ROC curve for calprotectin serum level for diagnosis of the severity of AV (mild and moderate versus severe).

\section{DISCUSSION}

Acne vulgaris is a chronic inflammatory skin disorder with complex pathogenesis. Hyperseborrhea, dyseborrhea, altered keratinization of the sebaceous duct, cutibacterium acnes colonization and inflammation have been proposed to play an essential role in the pathophysiology of acne. Furthermore, many hormones contribute to the pathogenesis of AV. These include androgens, insulins, and insulin-like growth factor-1. Moreover, hypersensitivity to androgen production and inflammatory cytokines affected by the innate immune system adds to the complexity of the underlying disease pathogenesis ${ }^{(\mathbf{1 1})}$.

Calprotectin, a calcium-binding protein, is a highly sensitive and objective marker of inflammation in many immunomodulatory, antimicrobial, and antiproliferative studies ${ }^{\mathbf{1 2}}$. The present study results revealed a highly statistically significant difference in serum calprotectin levels between the case group and the control group ( $\mathrm{P}$-value < .001). A highly significant positive correlation was also observed between serum calprotectin level and the severity of acne in the case group. The statistically significantly higher serum calprotectin levels in the AV group than the control group and the significant positive correlation between its levels and both disease duration and severity suggest that calprotectin can have a role in the pathogenesis of acne. This might be mediated by its possible role in the inflammatory process associated with AV.

The possible role of calprotectin in the pathogenesis of AV has been previously evaluated in one study only by Korkmaz and Fıçıcıoğlu ${ }^{(13)}$. They reported that serum calprotectin level in patients with AV was statistically significantly higher than that of the healthy controls and its level was higher in patients with moderate-severe acne than in those with mild disease. This comes in agreement with the present study results, reinforcing the proposed role of calprotectin in the pathophysiology of acne.

At the same time, the study results showed a significant positive correlation between serum calprotectin levels and the severity of acne. This is in agreement with Fouda et al. ${ }^{(14)}$ who reported similar results. However, this study results are in contrast with the results shown by Korkmaz and Fiçıcıoğlu (13) who reported no significant correlation between serum calprotectin level and severity of acne.

Measurement of fecal calprotectin is considered a reliable indicator of inflammation. Therefore, it has been used routinely and successfully to manage inflammatory bowel disease ${ }^{(15)}$. Furthermore, serum calprotectin, as a novel blood-based biomarker, has been the subject of many recent investigations, and it has been suggested that serum calprotectin may be more representative of systemic inflammation than intestinal inflammation ${ }^{(16) .}$

Studying the correlation between calprotectin and the grade of $\mathrm{AV}$ revealed a significant correlation $(\mathrm{P}<0.0001)$.

ROC curve analysis proved that calprotectin could act as an accurate predictor of acne development and may be an excellent tool for providing care and follow-up to patients with AV. Furthermore, calprotectin can provide a tool to assess acne case severity, either mild, moderate, or severe. However, the ability of calprotectin to distinguish between mild, moderate and severe cases was excellent. ROC Curve provides a useful way to evaluate the sensitivity and specificity for quantitative diagnostic measures that categorize cases into one of two groups ${ }^{(\mathbf{1 7})}$. 
This study demonstrated that serum calprotectin concentration with a cutoff point higher than $1.03(\mathrm{ng} / \mathrm{ml})$ had $77.5 \%$ sensitivity, $80 \%$ specificity, $79.5 \%$ PPV, $78 \%$ NPV and accuracy of $78.75 \%$ for AV diagnosis. While, for detection of AV severity using mild versus moderate and severe cases, the cutoff point higher than $1.1(\mathrm{ng} / \mathrm{ml})$ had $96.3 \%$ sensitivity, $100 \%$ specificity, $100 \%$ PPV, $92.8 \%$ NPV and accuracy of $97.5 \%$. While, using mild and moderate versus severe cases, the cutoff point higher than 1.22 (ng/ ml) had 92.9\% sensitivity, 100\% specificity, 100\% PPV, 96.3\% NPV and accuracy of $97.5 \%$. These data showed that serum calprotectin concentration can be a discriminator for the diagnosis of $\mathrm{AV}$ and detection of disease severity with high specificity and sensitivity of the test.

If the test is to be used in clinical practice, it must be reliable, with an acceptably low rate of falsepositive and/or false-negative results. To evaluate the performance of a test in detecting a disease in a given population, sensitivity, specificity, positive predictive value, and negative predictive value are the parameters to be calculated ${ }^{(\mathbf{1 8})}$.

Based on the present study finding, we propose that calprotectin may serve as a reliable marker of acne severity and can help evaluate the response to treatment. Furthermore, calprotectin might be a potential target for the treatment of acne based on its possible role in the mediation of the inflammatory process. However, it is very early to make a definite conclusion in this respect.

Limitations of the study were: Firstly, a relatively small sample size. Secondly, few inflammatory markers were investigated among many ones related to AV. Thirdly, it is well known that every disorder has many risk factors (genetic, environmental, racial...etc.). But, we tried to fix the race (Egyptians) with the age and sex categories. Fourthly, the absence of serum calprotectin evaluation after-treatment of the studied patients.

\section{CONCLUSIONS}

The study showed that serum calprotectin levels are increased in AV patients compared to healthy control subjects and are positively correlated with disease severity. Therefore, it might help evaluate the severity of AV. Further larger and well-controlled studies are recommended to emphasize the possible contribution of calprotectin in the pathogenesis of acne vulgaris and its role as a biomarker in evaluating disease severity and its response to treatment.

\section{REFERENCES}

1. Cong T, Hao D, Wen X et al. (2019): From pathogenesis of acne vulgaris to anti-acne agents. Archives of Dermatological Research, 311 (5): 337-49.
2. Dréno B, Pécastaings S, Corvec S et al. (2018): Cutibacterium acnes (Propionibacterium acnes) and acne vulgaris: a brief look at the latest updates. Journal of the European Academy of Dermatology and Venereology, 32 (1): 5-14.

3. Abd-Elmaged W, Nada E, Hassan M et al. (2019): Lesional and circulating levels of interleukin-17 and 25hydroxycholecalciferol in active acne vulgaris: Correlation to disease severity. Journal of Cosmetic Dermatology, 18 (2): 671-6.

4. Uslu G, Şendur N, Uslu M et al. (2008): Acne: prevalence, perceptions and effects on psychological health among adolescents in Aydin, Turkey. Journal of the European Academy of Dermatology and Venereology, 22 (4): 462-9.

5. Cheng T (2017): Calprotectin: a key and versatile protein. Hong Kong J Dermatol Venereol., 25 (1): 107- 108.

6. Wieland C, Vogl T, Ordelman A et al. (2013): Myeloid marker S100A8/A9 and lymphocyte marker, soluble interleukin 2 receptor: biomarkers of hidradenitis suppurativa disease activity?. British Journal of Dermatology, 168 (6): 1252-8.

7. Wang S, Song R, Wang Z (2018): S100A8/A9 in Inflammation. Front Immunol., 9 (1): 1298-303.

8. D'Amico F, Granata M, Skarmoutsou E et al. (2018): Biological therapy downregulates the heterodimer S100A8/A9 (calprotectin) expression in psoriatic patients. Inflammation Research, 67 (7): 609-16.

9. Thorey I, Roth J, Regenbogen J et al. (2001): The Ca2+binding proteins S100A8 and S100A9 are encoded by novel injury-regulated genes. Journal of Biological Chemistry, 276 (38): 35818-25.

10. Kim J (2005): Review of the innate immune response in acne vulgaris: activation of Toll-like receptor 2 in acne triggers inflammatory cytokine responses. Dermatology, 211: 193-198.

11. Tuchayi S, Alexander T, Nadkarni A et al. (2016): Interventions to increase adherence to acne treatment. Patient Preference and Adherence, 10 (2): 2091-98.

12. McNeill E, Hogg N (2014): S100A9 has a protective role in inflammation-induced skin carcinogenesis. Int $\mathrm{J}$ Cancer, 15 (4): 798-808.

13. Korkmaz S, Fıçıcıoğlu S (2018): Calprotectin can play an inflammatory role in acne vulgaris. Postepy Dermatologii i Alergologii., 35 (4): 397-399.

14. Fouda I, Obaid Z, Hegazy S et al. (2021): Calprotectin in acne vulgaris: A possible contributory role. Journal of Cosmetic Dermatology, 20 (2): 621-5.

15. Zhang M, Zhang X, Zhang J (2016): Diagnostic Value of Fecal Calprotectin in Preterm Infants with Necrotizing Enterocolitis. Clin Lab., 62 (5): 863-869.

16. Kopi T, Shahrokh S, Mirazi S et al. (2019): The role of serum calprotectin as novel biomarker iniflammatory bowel diseases: a review study. Gastroentrol Hepatol Bed Bench., 12 (3): 183-189.

17. Hazarika N, Archana M (2016): The psychosocial impact of acne vulgaris. Indian Journal of Dermatology, 61 (5): 515-520.

18. Oreide K (2009): Receiver-operating characteristic curve analysis in diagnostic, prognostic and predictive biomarker research. J Clin Pathol., 62: 1-5. 